\title{
Link Budget Design with Performance Evaluation of Tunable Impulse-Based Ultra Wideband to Support the Integration of Wireless Sensing and Identifications Infrastructures
}

\author{
Mohammed Seed Jawad, Widad Ismail, Ayman Hajjawi, Othman Abdul Rani, \\ Azahari Saleh \\ Faculty of Electronics and Computer Engineering (FKEKK), Univeristi Teknikal Malaysia Melaka (UTeM), Durrian \\ Tunggal, Malaysia \\ Email: saeed@utem.edu.my, eewidad@usm.my, ayman_2747@yahoo.com, rani@utem.edu.my, \\ azahari@utem.edu.my
}

Received 13 June 2014; revised 14 July 2014; accepted 13 August 2014

Copyright (C) 2014 by authors and Scientific Research Publishing Inc.

This work is licensed under the Creative Commons Attribution International License (CC BY).

http://creativecommons.org/licenses/by/4.0/

(c) (i) Open Access

\begin{abstract}
Recently, many studies propose the use of ultra-wideband technology for passive and active radio frequency identification systems as well as for wireless sensor networks due to its numerous advantages. By harvesting these advantages of IR-UWB technology at the physical-layer design, this paper proposes that a cross layer architecture platform can be considered as a good integrator for different wireless short-ranges indoor protocols into a universal smart wireless-tagged architecture with new promising applications in cognitive radio for future applications. Adaptive transmission algorithms have been studied to show the trade-off between different specific QoS requirements, transmission rates and distances at the physical layer level and this type of dynamic optimization and reconfiguration leads to the cross-layer design proposal in the paper. Studies from both theoretical simulation and statistical indoor environments experiments are considered as a proof of concept for the proposed architecture.
\end{abstract}

\section{Keywords}

Radio Frequency Identification (RFID), Ultra-Wideband (UWB), Impulse-Based Ultra-Wideband (IR-UWB), Cognitive Radio (CR), Time Hopping Pulse Position Modulation (TH-PPM), Quality of Service (QoS) 


\section{Introduction}

The radio frequency identification (RFID) technique, as a form of wireless communication system, is currently gaining more interest in the academia and in industries. Its emerging and promising applications, mainly in logistics, health care, military, and warehouses, can significantly reduce cost, improve productivity, and help users achieve real-time tracking. Recent tag standards, especially for passive tags, use narrow band radio signals to provide a signal that is strong enough to power-up the tags chips and establish a communication link between the tags and the readers. This narrow band signal is received as a backscattered signal at the reader [1]. Many research and literature now focus on the wide deployment of this technology. Compared with the barcode system, the optimum level of deployment of this technology has not yet been reached. Most of the recent studies address three related problems. First is the security problem. As RFID applications have become increasingly prevalent in daily life, privacy and security issues become more critical for individuals and organizations. Second is the integration problem with other wireless technologies. Since there is a lack of maturity and highlevel integration with RFID and other wireless networks, such as Wi-Fi, WSNs and WAN, this causes the actual shortfall of industrial adoption. Finally and perhaps most importantly, is the problem of hardware cost for RFIDs' tags and readers for widely deployment of this technology. Many believe that, the tag circuit should be as low price as a few cents only.

RFID and WSN having different functionalities as the first one is mainly supporting detection and identification of the objects, while the second sensing objects and environmental changes, processing these data and transmit them. At the same time, both technologies share some common design constraints at the hardware level, since the wireless node or tag should be as low cost with very low power consumption to achieve the widely deployment of these technologies. Integrating both technologies in one network infrastructure can combine both functionalities of wireless detection and sensing which will open new domains for future application in digital smart environments such as digital smart homes, hospital, warehouses and others.

Ultra-wideband (UWB)-based RFID tag technology shows good features at the wireless signal-level as well as at the hardware implementation level [2]. Generating UWB wireless signal for communication can be considered secure wireless communication physically; this means that it is infeasible for any intruder to intercept the signal. It can also be categorized under low duty cycle (low power signal), which shows good resistance to narrow band inference. The advantage of generating low duty-cycle pulses in a wide-band is that, the signal shows the good immunity level in multi-path fading channel. Since the physical layer can generate secure wireless signal at the hardware level, it eliminates the need to implement traditional encryption algorithms at the medium access control (MAC) layer, and this leads to more simplified hardware architecture with no need for extra logic gates required for encryption to secure the data contents. This is usually in typical and recent cases done at the MAC layer level. The objectives of applying algorithms for securing the data content and avoiding signal collision at the MAC layer design level can be directly accomplished through the physical layer reducing computation and hardware complexities [3].

Impulse-based ultra-wideband radio technology (IR-UWB) for low data rates wireless sensors, can be utilized for future radio frequency identifications (RFID) and wireless sensor network (WSN) architectures [4] and can be considered as good integrator for both of them. This recent technique demonstrates the advantages of achieving higher data rates, facilitating the possibility of operation ranges to reach a few hundred meters with more accurate position estimation (centimeter accuracy), incurring very low energy per bit with low cost of hardware implementation. Aside from the adaptive properties of the IR-UWB, this technique also satisfies the requirements for non-interference with other licensed systems. The adjustable parameters can support different data rates (by tuning the parameters of different modulations and signal shapes) and different spectrum occupation (by adjusting the center of the frequency and the bandwidth), moreover, this technique ensures adaptive multiple access and security of information. All these advantages of IR-UWB over WSN and RFID integrating both of them in one infrastructure consider as a good candidate for future cognitive radio nodes [5]-[8]. The main contribution of this paper is, proposing IR-UWB cross-layer architecture capable of performing wireless sensing and identification in short to medium wireless indoor communication scenarios, at the same time supporting different data rates and QoS requirements by the ability of self-adjusting its parameters according to the level of QoS dropping at the dynamic indoor environments. The focuses here are mainly at the physical layer level to prove the concept of tunable generated signal in point to point communication according to optimization criteria in Data-Link layer to reconfigure the signal transmission parameters to be optimized. However the optimization algorithm at the MAC layer is proposed in this paper whereas the optimum parameters selection for certain QoS 
requirement is out of the scope of this paper and such optimum selection can be done using genetic algorithm to generate a population for the most optimum parameters according to the optimization criteria suggested in this paper. With this cross-layer architecture, the wireless nodes are able to interact with each other's in dynamic channel conditions and for different applications requirements. The MAC layer (or the Data-Link layer) has the capability of evaluating the performance according to the BER Threshold representing of QoS requirements for certain wireless applications, then it will send reconfiguration signal to instruct the physical layer to tune its parameters according to the new situations. Time hopping pulse position modulation (TH-PPM) scheme had been chosen due to its simplicity and suitability to meet the design constraints of wireless sensing and identifications nodes. Signal model and physical layer specifications will be discussed in detail in Section 2. Adaptive UWB TH-PPM system architecture and the steps in tuning the physical layer parameters to meet the requirements of QoS will be discussed in Section 3. The QoS will be evaluated as bit error rate (BER) performance, for the relationship between the different data rates traffics and different operational ranges coverage. The last section will show some simulated results as a proof of concept of the proposed architecture. Then the work will be concluded for future research.

\section{Previous Studies}

In literature, many published research works proposing IR-UWB as alternative physical layer for wireless network technologies mainly for indoor applications [3] [9] and [10]. Most of the applications of this technology utilized for wireless sensors for ranging, positioning and motion detection for realistic multi-path fading environments these works have been done in simulating the IR-UWB sensors performance in indoor fading channels models or by setting real data experiments for some indoor communication scenarios, these can be found in researches referred in [11]-[13], but many of them are outdated or missing comprehensive studies especially about understanding the fully optimization of implementing IR-UWB as alternative physical layer. some aspects of these limitations can be understood as: The UWB technology in many proposed scenarios is not fully utilized over the wireless indoor network infrastructure, since they assumed from the beginning the UWB technology is applied only as upper link communication (from the tags to the readers) where the downlink is still narrowband (to power-up the tags using the energy harvested from the backscattered narrow band signal) as a result of that, this will increase the overall tag's and system's complexity since the two link communication are different as one is UWB communication and the other narrowband communication. Another point of limitation, that its widely believed that applying IR-UWB as alternative physical layer will decrease the load in MAC layer design as a straightforward consequences of the good characters of the generated pulse signal, since these type of signal is in general described as, secure (infeasible to detect), immune to multipath fading (due to low duty cycle signal spread over wide or ultra wide band) and resistant to interferences especially interference to narrowband signal (since the signal very low power and near to noise level), but the focus has been more on MAC layer level rather than the physical layer level and signal design.

Researches as referred in [14] [15], propose the Integration of RFID and WSNs wireless infrastructures using Impulse-based UWB technology. Many models of the integration architectures have been proposed and investigated depending whether the reader is RFID reader and combined with sensor for two RFID and WSN heterogeneous networks or reader itself having the capabilities of sensing and identification as one device sensing the object location and the channel conditions.

The limitation of such researches is that, most of them focusing on combing both RFID reader and the wireless sensor with two different wireless protocols, rather than making the reader itself capable of performing wireless identifications, sensing and detection at the same time in one platform and this is where here this research trying to address and investigate in brief by introducing alternative physical layer suitable for wireless sensing, identification and detection by optimizing the Impulse-based UWB signal, with Time hopping modulations techniques such as (TH-PPM \& PAM), these modulations techniques are carefully been chosen among other possible UWB techniques, since they suitable for low to medium data rate wireless indoor infrastructures such as FRID and WSNs also low hardware complexity which meet the design constraints especially for the RFID tags.

\section{IR-UWB Signal Model}

The main point of choosing Pulse-Position modulation as a coding technique for the UWB generated short 
pulses train is that, this modulation technique is simple and good enough and suitable for Ultra-low power RFID and WSN nodes [1] [7]. The TH $M$-ary (different binary orders) with PPM modulation for $k$-th user can be given by [8]:

$$
S^{K}(t)=\sum_{J=-\infty}^{\infty} A^{(k)} d_{\left[J / N_{s}\right]} p\left(t-T_{f}-C_{J}^{(k)}-\delta d_{\left[J / N_{s}\right]}^{(t)}\right)
$$

where $S^{K}(t)$ is the transmitted TH-PPM signal for $K$-th user, $P(t)$ represents pulse shaping, which is the second derivative of Gaussian pulse with pulse width $T_{p} ; T_{f}$ is the frame time and each frame is divided into $N_{h}$ time slots with duration time $T_{c} ; N_{s}$ is number of pulses per bit and $C_{j}^{(k)}$ is the pulse shaping patterns, which are pseudorandom numbers with period $T_{c}$ representing the time hopping sequence for $K$-th user. Additional shift is required to avoid catastrophic collisions caused by the multiple access interference (MAI). The $d$ parameter is the sequence for the data stream generated by the $K$-th user after channel coding. $\delta$ is the shift introduced for pulse position modulation utilized by $M$-ary PPM. Table 1 as well shows all these transmitted signal parameters with their typical values.

If it is assumed that the signal amplitude $A^{(v)}=1$ for the $M$-ary PPM signal, then Equation (1) can be written as [9] [16]:

$$
S^{(v)}(t)=\sum_{J=-\infty}^{\infty} p\left(t-j T_{f}-C_{j}^{(k)} T_{c}-\delta d_{\left[J / N_{s}\right]}^{(k)}\right) .
$$

Figure 1, summarizing IR-UWB signal's parameters with the description for each parameter and its typical values, while in Figure 2 simulation shows the generated signal according to the above mentioned parameters.

The model for the received signal $r(t)$ for $k$-th users can be written as:

$$
r(t)=\sum_{k=1}^{k=n}\left(S^{(k)}\left(t-\tau_{v}\right)\right)+n(t)
$$

where $s(t)$ is the transmitted signal after $n(t)$ noise is added, and delayed by time delay $\tau_{v}$.

\section{UWB Wireless Tagged-Based Communication}

The following are the summarized steps for generating UWB TH-PPM signal and the demodulation at the receiver front-end cab as Figure 2 also showing:

1) Initially powering up the tag: The tag here is active or semi active tag powered by long life time battery to generate the IR-UWB signal.

2) Communication: The tag sends its unique ID, stored in its memory to the reader. This uplink communication adopts UWB with TH-PPM scheme.

\begin{tabular}{ccc}
\multicolumn{2}{l}{ Table 1. UWB TH-PPM signals parameters. } & Description \\
\hline Signal parameter & Value \\
\hline $\begin{array}{c}\text { Power } \\
f_{c}\end{array}$ & Sampling frequency (Hz) & $-30 \mathrm{dBm}$ \\
Numbit & Number of bits generated by source & $50 \mathrm{e}$ \\
$N_{s}$ & Number if time the pulse is repeated & 2 \\
$T_{f}$ & Frame time (s) & 5 \\
$T_{c}$ & Chip time (s) & $3 \mathrm{e}-9$ \\
$N_{h}$ & Periodicity of the TH code & $1 \mathrm{e}-9$ \\
$N_{p}$ & The time duration for each pulse & 3 \\
$t_{m}$ & Shaping factor of the pulse & 5 \\
tau & Time shift caused by PPM modulation & $0.5 \mathrm{e}-9$ \\
$\delta$ & & $0.25 \mathrm{e}-9$ \\
\hline
\end{tabular}




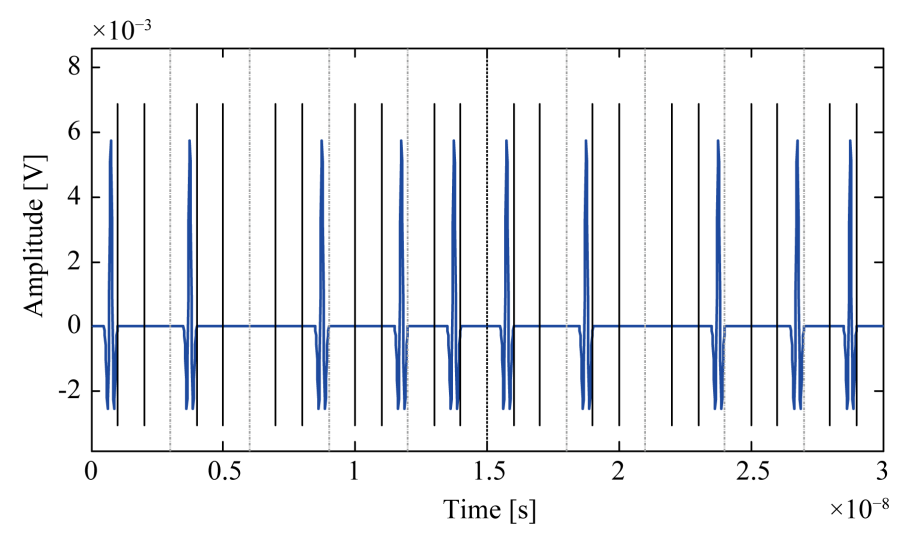

Figure 1. Simulations for generated TH-PPM signal.

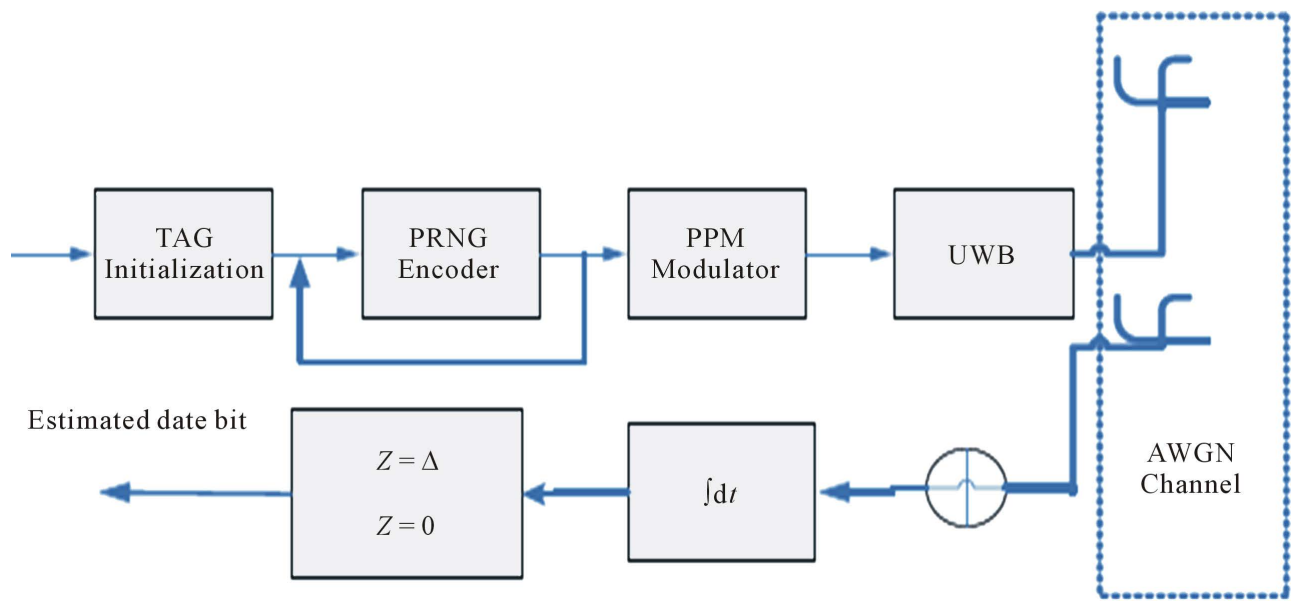

Figure 2. UWB TH-PPM communication system model.

3) A pseudo-random generator (PRNG): as spectrum spreading encoder, this is where the time slot of the pulses is coded; these sequences are considered as secret keys and will only be known for the tag and reader. The tag's memory after each read cycle stores the sequence of the last number generated by the PRNG. When another readout cycle starts, the PRNG sets another pseudo-random modulation, depending on the previous stored codes in the memory. This adds more security and makes system attacks more difficult.

4) Demodulation phase: to demodulate the incoming UWB signal and get the original narrowband signal, the receiver should correlate the incoming signal pulses with a template signal, since the slots' time sequences for the incoming pulses are known beforehand. Two correlation operations should be done, one at $t=0$ to expect the logic 0 and another correlation at $t=\delta$ expecting logic 1 . One of the correlations will capture the received signal.

5) Acquisition: Its responsible for locking the receiver to a transmitter's signal; this can be accomplished by setting the acquisition portion of the packet with certain code length (e.g. 16 pulses), configured and defined by the end-user, so once the threshold settings for the acquisition met, the transmitter and the receiver are code aligned and roughly frame aligned [8] [17]. The overall system architecture based on UWB previous modulation and demodulation concepts is shown in Figure 3.

\section{Adaptive UWB Tags}

IR-UWB can be categorized as a wireless indoor technology. The data rate can be varied for different operational ranges and applications. For example, the medical-based sensors nodes that form wireless body sensor network (WBAN) can be considered as having a high data-rate with very short-range, whereas the IR-UWB used for RFID wireless systems can be considered as having a low data rate with short-to-medium operational range. 


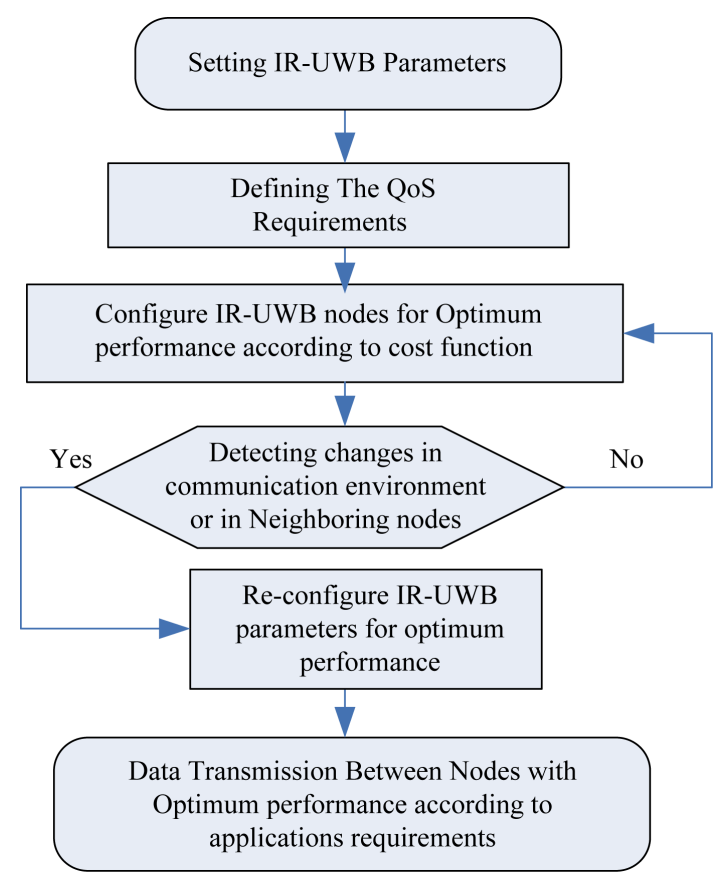

Figure 3. Flowchart illustration of the adaptive IRUWB transceiver.

In this case, when the distance increases while the data-rate kept fixed, the BER performance will be degraded. To meet the quality of service constraints under multiple access environment and requirements for IR-UWB systems, the UWB-physical layer should efficiently adapt a number of parameters that meet the QoS requirements. The QoS can be evaluated as the BER performance under an adaptive white Gaussian noise and multipleaccess communication [18]. Fortunately, a wide range of UWB parameters, such as pulse duration $\left(T_{m}\right)$ number of pulses per information $\left(N_{s}\right)$, number of chips $\left(N_{c}\right)$, and modulation type [19] can be adjusted at the physical layer design, allowing for a highly adaptable UWB system. The system can be matched for a wide range of applications and services using this unique functionality. This adaptive approach can support multi-rate transmissions based on the QoS requirements and the channel state information (CSI).

\subsection{IR-UWB in Adaptive Sensor Tag}

This section defines a local cost function in each IR-UWB transceiver node to optimally and dynamically allocate appropriate resources to efficiently meet the QoS requirements. The QoS requirements include the data rate, BER and the energy dissipation. The resources allocations are the possible values of PRI (Pulse Repetition Interval) and $m$ (Modulation Binary Order) and the combination of these two parameters define the modulation scheme which affect the data rates and the required Eb/No ratio (where Eb is the bit energy and No is the noise level).

Nodes transmit data with the most efficient modulation scheme. Also, the initiating node extracts the suggested modulation scheme from the control packet of clear to sent (CTS) that allow the multiple access by CSMA/CA (Carrier Sense Multiple Access/Carrier Access) or, from the beacon of a PNC (Point Network Coordinator) of IEEE 803.15.3.

The adaptive system consists of a transmitter, receiver, physical layer, MAC layer, application layer and control unit. Other network layers that do not interact with the control unit are not considered. The control unit easily generate various $M$-ary modulation schemes and PRI values for different data rates transmissions.

Different types of interferences affecting IR-UWB system. These interferences are: co-channel interference which is happened in a network when a system capture the intended signal while one or more addition IR-UWB system transmit simultaneously. Inter-symbol interference (ISI) which is caused by the delayed multipath from the previous symbol interference. Also, intera-symbol interference as a result of a multipath from current symbol 
which cause a higher correlation from the first multipath which leads to misinterpretation.

As a conclusions from different literature studies about the effects of interferences on UWB systems in literature [19]-[21], ISI increases as PRI decreases and the effect of intra-symbol interference and co-channel interference increase as $m$ increase. Thus, settings of high $m$ and short PRI are more susceptible to interference, which result in limitation on performance regardless of Eb/No. Alternatively, low $m$ and long PRI are less susceptible to interference, and thus the performance limitation is more to Eb/No ratio. Based on that, in low Eb/No ratio a high $m$ are preferable and in higher Eb/No ratio (where the interferences dominates the noise), a modulation scheme with low $M$ are preferable.

The local cost function

$$
P=\frac{\mathrm{BER} \cdot \mathrm{Eb} / \mathrm{No}}{\text { Data rate }}
$$

[22], where $P$ is the performance evaluation.

The BER is a function related to $m$ (modulations types), PRI (pulse repetition interval), Eb/No (average bit energy), channel impulse response $h(x)$, and interference $i(x)$; and is expressed as

$$
\mathrm{BER}=W(\mathrm{~Eb} / \mathrm{No}, \mathrm{PRI}, m, h(t), i(x))
$$

$\mathrm{Eb} / \mathrm{No}$ is a function related to the maximum distance between the transmitter and the receiver, as well as the maximum radiated power allowed for a given data rate, which can be expressed as

$$
\mathrm{Eb} / \mathrm{No}=Y(\text { dist }, m, \mathrm{PRI}) \text {. }
$$

The data rate depends on both $m$ and PRI

$$
\text { Data Rate }=\left(\log _{2} m\right) / \text { PRT. }
$$

Thus from Equation (4) to (7), $P(z)$ define the local cost function, where $z$ is a set of environmental QoS 6-parameters $[\mathrm{Eb} / \mathrm{No}, m, \mathrm{PRI}, h(x), i(x)$, dist $]$ within $Z$, the set of all possible 6-parameters. Thus

$$
P=\frac{\text { PRI } \cdot w(\text { Eb } / \text { No, PRI, } m, h(x), i(x)) \cdot Y(\text { dist }, m, \text { PRI })}{\log _{2} m} .
$$

As the environmental conditions or QoS requirements vary, the local cost function defined as local expected cost as in Equation (48) for a given probability distribution of

$$
\bar{P}=\sum_{\forall \in Z} P(z) \cdot P_{\text {Previous }}(z) .
$$

Optimization of the local cost function is based on the choice of modulation scheme in relative of the global cost function which is sum of all local cost functions in the network. Therefore, optimized configuration can be achieved if the local IR-UWB node has less cost than other possible configurations for certain environment and QoS parameters.

\subsection{Cross-Layer Design Architecture}

Fixing the IR-UWB parameters in order to meet the QoS requirements for multiple-access IR-UWB system is inadequate for operational RFID systems' ranges and services. Therefore, the adaptive IR-UWB architecture seems to be a good solution to meet the required QoS. The adaption scheme can be done according to the following proposed steps: First, all the IR-UWB transmitter node transmits bits at regular intervals, and then the reader listens and calculates the BER to evaluate the performance. Second, if the BER calculations at the reader do not match the satisfactory level for the required QoS (this is done by proposing the weighing algorithm at the MAC layer level) for that specific application and operational range. Then; the reader instructs the transmitter nodes (RFID-Tags) to adjust its parameters to meet the requirements. Figure 4, showing the architecture design of the proposed system. The trade-off flexibility of IR-UWB for different date-rates, operational-ranges for specific BER requirements is simulated.

In Figure 5, performance simulation confirming that, the BER performance can be enhanced by tuning the time hopping different modulations techniques.

The BER performance can be set as pre-determined QoS requirements. In varied distances scenarios, the BER 


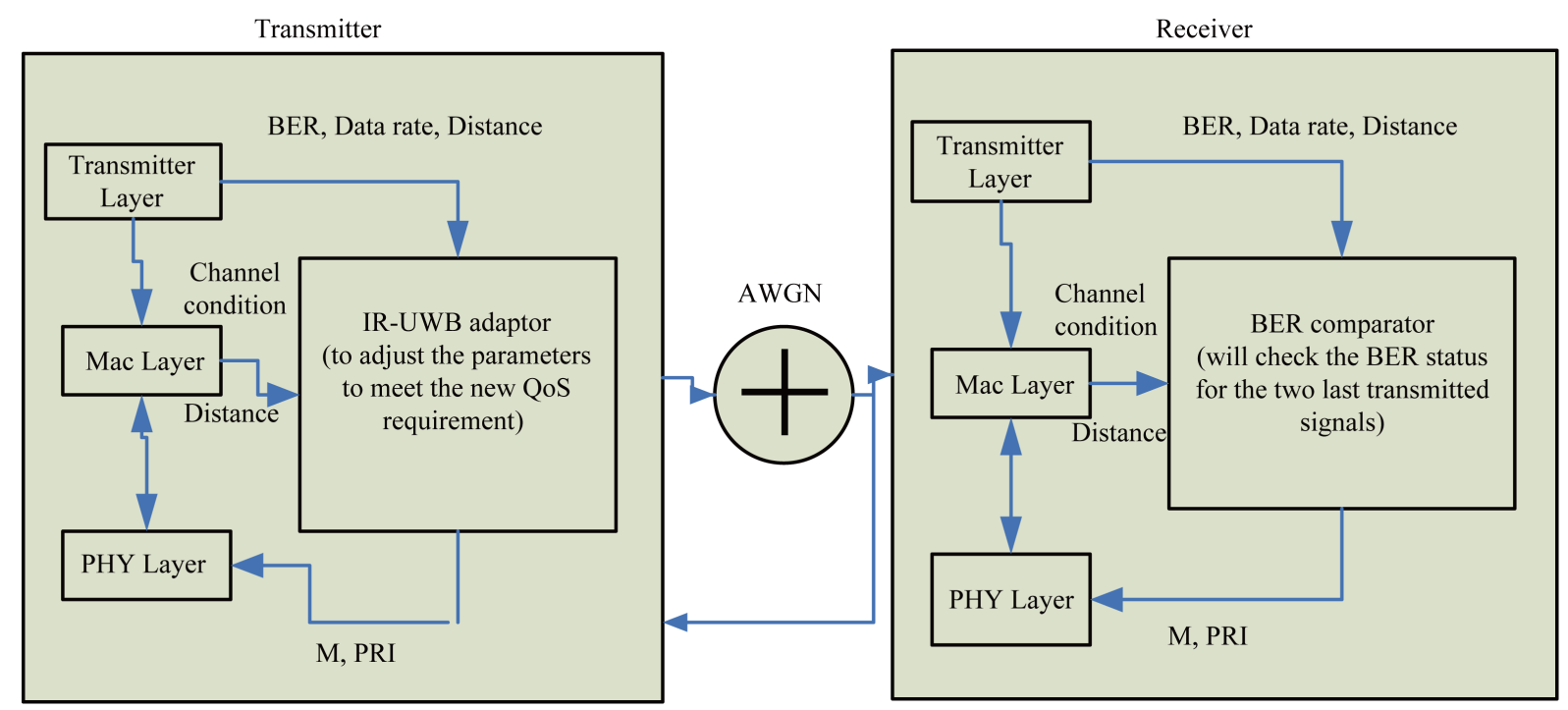

Figure 4. Proposed architecture for adaptive UWB-RFID communication model.

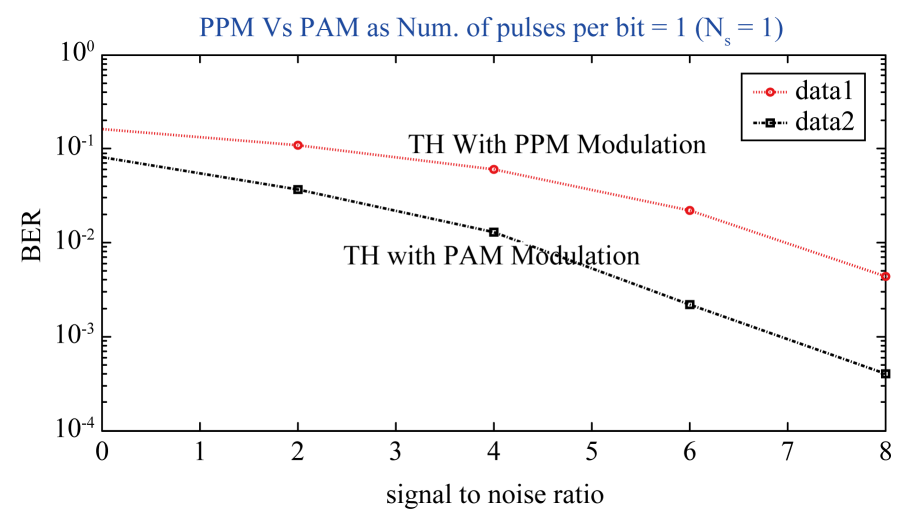

Figure 5. BER performance comparison for PPM vs PAM TH-UWB.

can be tuned to certain level to meet the required performance as the following Figure 6 shows.

\section{Simulated and Experimentally Analyzed Results}

Simulations are carried out to show the different parameter settings for IR-UWB. Fortunately wide range of parameters at the physical layer design level including parameters for signal transmission, signal coding and pulse shaping can be tuned to meet the requirements of UWB-RFID systems. First, the generated binary data (transmitted signal), the output of the uniform random number generator, is modulated by pulse position modulation. The generated AWGN noise is then added (multi-path fading channel model noise) to the modulated signal. At the receiver part, the pilot generates the template signal and then the demodulated signal is constructed. Simulations are carried out for different numbers of parameter settings in real-time Digital Signal Possessing (DSP) Simulation environment as prove of concept. These parameters as shown in Figure 7 can be adjusted to change the data rate, assuming that we have specific QoS requirements for different operational ranges and data traffic.

Table 2, show a fixed-point real-time simulation results for the whole communication link of the adaptive IR-UWB is presented to show in real-time simulation the generated signal, its propagation behavior on AWGN and multipath fading channel model. The received and the modulated signal at the receiver side, with also the option of displaying the BER calculation as the simulation on.

The results of the measurements conducted in selected indoor under NLOS conditions, wherein numerous thick and thin walls, electrical equipment, and the distances between the transmitters are considered. The layout of this test site is shown in Figure 8, where $T_{1}$ represents fixed transmitter location; and $R_{1}, R_{2}, R_{3}$, and $R_{4}$ 


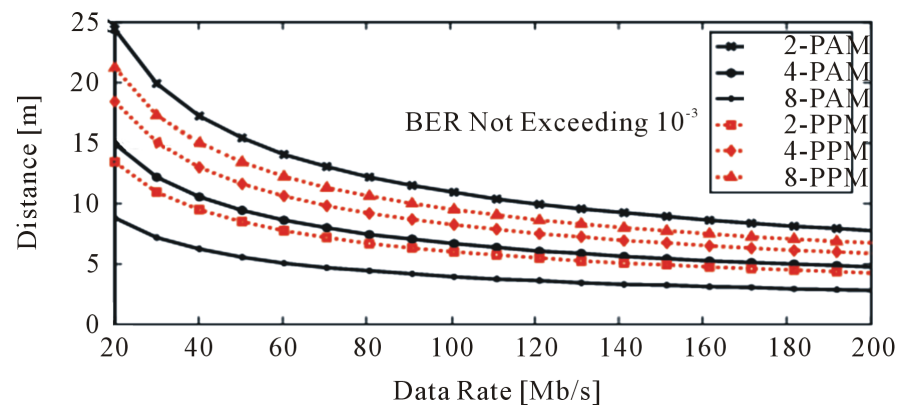

Figure 6. Trade-off flexibility evaluations as a function of the data rate based on different pulses modulations.

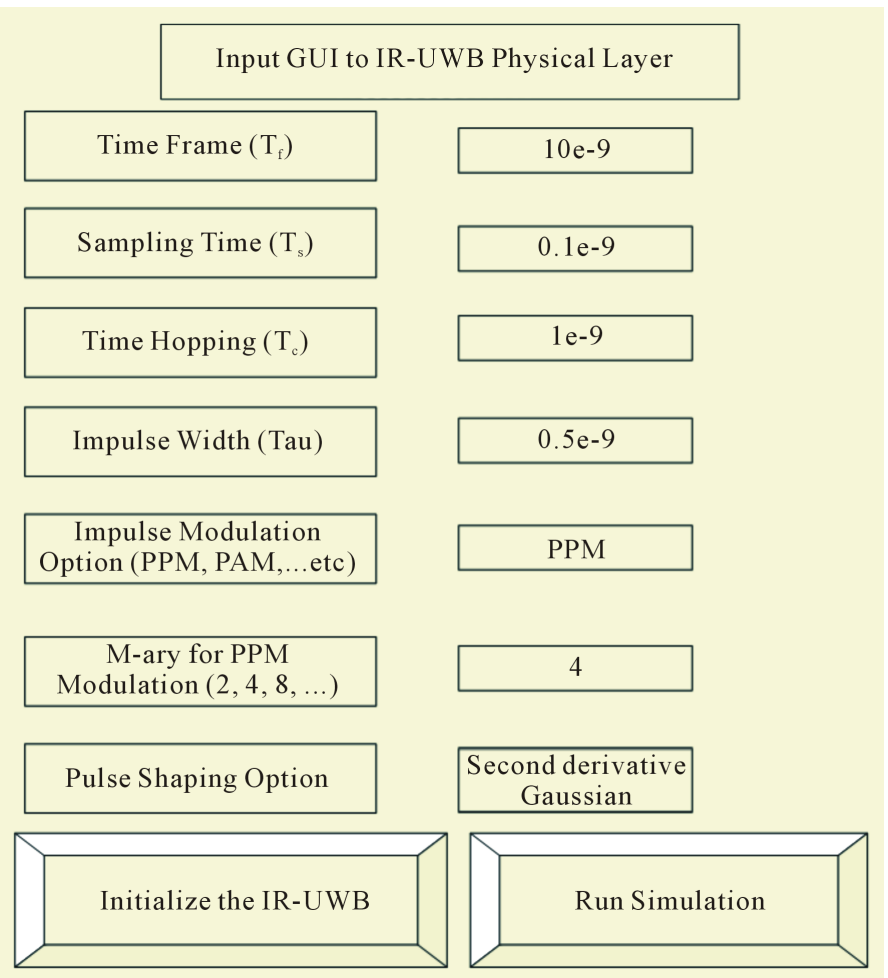

Figure 7. Graphical user interface showing different IR-UWB PHY parameter settings.

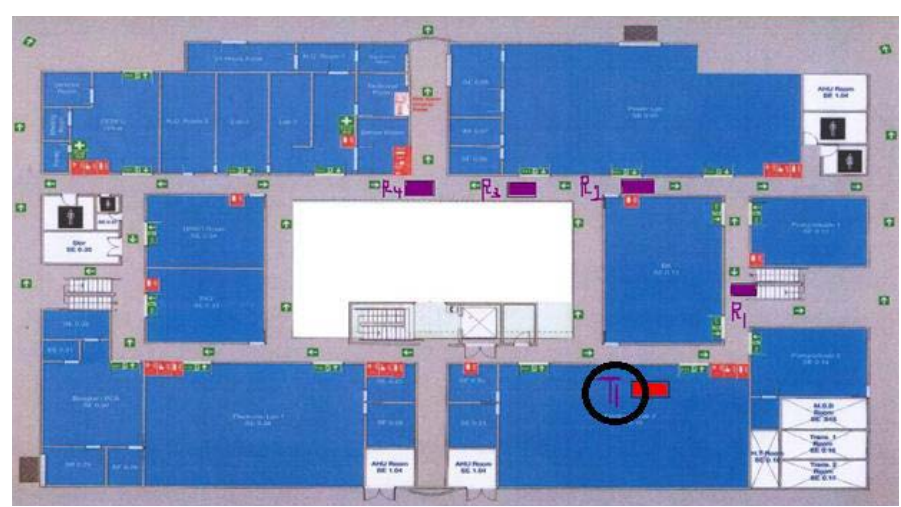

Figure 8. Experimental layout of indoor propagation. 
Table 2. IR-UWB system parameters and evaluation in real time.

\begin{tabular}{|c|c|}
\hline \multicolumn{2}{|l|}{ Data source } \\
\hline$M$-ary & 2 \\
\hline Symbol time & $100.000 \mathrm{~ns}$ \\
\hline Link selector number [0 reserved for pilot] & 1 \\
\hline Promised data-loading state & [11000] \\
\hline \multicolumn{2}{|l|}{ Modulation } \\
\hline Frame time & $10.000 \mathrm{~ns}$ \\
\hline TH unit time & $1.000 \mathrm{~ns}$ \\
\hline PPM unit time & $0.156 \mathrm{~ns}$ \\
\hline Impulse width & $0.2877 \mathrm{~ns}$ \\
\hline Symbol time in terms of frame time $T / T_{f}$ & 10 \\
\hline PN sequence period & 31 \\
\hline Average signal power & $-40.0 \mathrm{dBm}$ \\
\hline Pulse energy & $1.000000 \mathrm{e}-015 \mathrm{~J}$ \\
\hline Initial state of PN code shift register $[m=5]$ & [10001] \\
\hline Bits used to generate hopping code & 3 \\
\hline \multicolumn{2}{|l|}{ Channel } \\
\hline \multicolumn{2}{|l|}{ IEEE 802.15.3a channel model } \\
\hline UWB signal detection threshold & $95 \%$ \\
\hline Acquisition threshold & $70 \%$ \\
\hline False alarm threshold & $30 \%$ \\
\hline Initial state of receiver PN code shift register & {$[00010]$} \\
\hline \multicolumn{2}{|c|}{ Simulation in real time } \\
\hline \multicolumn{2}{|c|}{$\begin{array}{c}\text { Sampling time }\left(\text { uwb. } T_{s}\right)=0.0500 \mathrm{~ns} ; \text { UWB signal detected; } \\
\text { Time }=4.550 \mathrm{~ns} \text {; coefficient }=97.515 \\
\text { Acquisition coefficient }=10.092 \% \\
\text { Acquisition coefficient }=-3.805 \% \\
\text { Acquisition coefficient }=100.319 \% \\
\text { PN hopping code acquisition } \\
\text { Acquired frame time }=590.000 \mathrm{~ns} \\
\text { Tracking coefficient }=101.779 \% \\
\text { Tracking coefficient }=100.507 \% \\
\text { Data synchronization; demodulation begins } \\
\text { Demodulation start time }=810.000 \mathrm{n}\end{array}$} \\
\hline BER command & Bercal \\
\hline BER result & $0.020000000000000 \mathrm{~s}$ \\
\hline
\end{tabular}

denote different receiver distances from the transmitter location. The estimated ranges between the four positions of the transmitter and the receiver are: $30.48 \mathrm{~m}, 38,1 \mathrm{~m}, 45.72 \mathrm{~m}$ and $60.96 \mathrm{~m}$.

The tunable impulse based UWB proposed solution is evaluated experimentally for the actual achieved data rate in different indoor distances as shown in Figure 9. It can be considered as map to check the performance of the designed solution at certain distance. In general, the throughput decreases as distance increases but, the throughput reduction is more obvious for high data rates in extreme distance. The throughput for indoor environment is efficient at low-to-medium data rates ranging from $300 \mathrm{Kbps}$ to $1.2 \mathrm{Mbps}$. By contrast, for high data rates ranging from 4.8 Mbps to 9.6 Mbps, the throughput is not supportive during the transmission of the output 


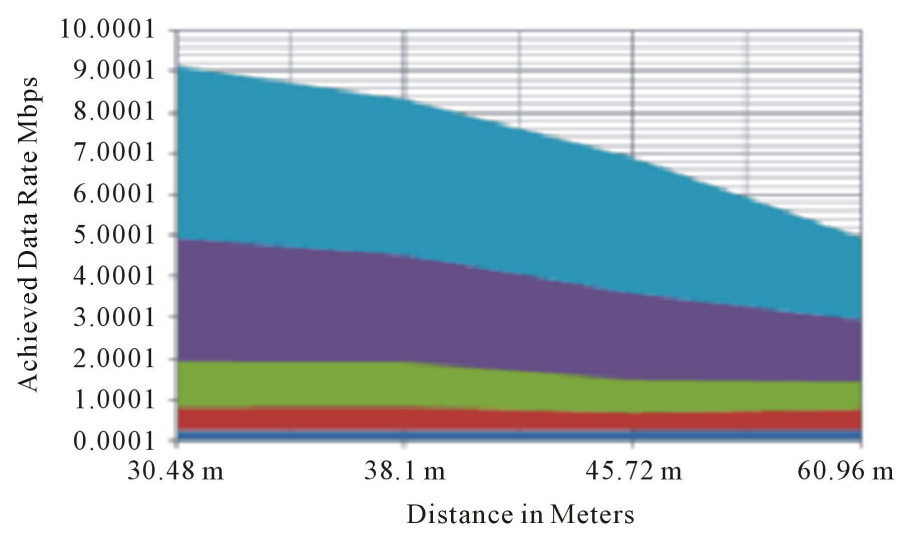

Figure 9. Throughputs (achieved data rate) as a function of distance between $T_{X}$ and $R_{X}$ in indoor radio environment.

power level of $-12.2 \mathrm{dBm}$ (approximately 50\% packet drops). At an extreme distance of 61 meters, the throughput decreases significantly, as higher data rate is more exposed to interferences and multipath effects. Also, the signal power attenuated due to the extended ranges.

\section{Conclusion}

In this paper, we proposed an adaptive UWB-RFID physical layer. By tuning parameter settings for IR-UWB, we can support different QoS requirements (BER and Data rate) for different operational ranges with location sensing capabilities and sensing for dynamic channel changes. The main advantage of the proposed architecture is its ability to support more wireless short to medium ranges indoor applications, with high to low data rates. With the ability of sensing the objects and the channel conditions, this system can integrate both RFID and WSN networks in cognitive radio scenarios for digital smart environments, by optimizing the TH-PPM ultra-wideband signal design. From the simulated and experimentally analyzed results, it's clearly shown that the careful selection of TH-PPM UWB signal parameters at the physical layer, will allow different data rates of the wireless transmission, allowing the system to response for the channel and the object's changes.

\section{Acknowledgements}

The authors would like to express their deepest gratitude and appreciation for Malaysia Ministry of High Education (MOHE) for the financial support under the grant coded as the following: ERGS/2013/FKEKK/TK02/ UTEM/03/05/E00021. As this finical support allows this work to be completed and published.

\section{References}

[1] Baghaei-Nejad, M., Zou, Z., Tenhunen, H. and Zheng, L.-R. (2007) A Novel Passive Tag with Asymmetric Wireless Link for RFID and WSN Applications. IEEE International Symposium on Circuits and Systems, New Orleans, 27-30 May 2007, 1593-1596.

[2] Lecointre, A., Dragomirescu, D. and Plana, R. (2008) Design and Hardware Implementation of a Reconfigurable Mostly Digital IR-UWB Radio. Romanian Journal of Information, 11, 295-318.

[3] Ha, D.S. and Schaumont, P.R. (2007) Replacing Cryptography with Ultra Wideband (UWB) Modulation in Secure RFID. IEEE International Conference on RFID, Grapevine, 26-28 March 2007, 23-29.

[4] Pezzin, M., Denis, B., Guillermin, R. and Piaget, B. (2007) A LDR IR-UWB Demonstration Platform for Services Based on Location and Tracking. 16th IST Mobile and Wireless Communications Summit, Budapest, 1-5 July 2007, $1-5$.

[5] Arslan, H. and Şahin, M. (2007) UWB-Based Cognitive Radio Networks. Cognitive Wireless Communication Networks, 213-230.

[6] Reed, J.H. (2005) An Introduction to Ultra Wideband Communication Systems. Prentice Hall, Upper Saddle River.

[7] Nejad, M.B. (2008) Ultra Wideband Impulse Radio for Wireless Sensing and Identification. Electronic and Computer Systems, KTH-Royal Institute of Technology, Stockholm. 
[8] Winy, M.Z. and Scholtz, R.A. (2006) Impulse Radio: How It Works. IEEE Communications Letters, 2, 36-38. http://dx.doi.org/10.1109/4234.660796

[9] Arslan, H., Chen, Z.N. and Di Benedetto, M.-G. (2006) Ultra Wideband Wireless Communication. John Wiley \& Sons, Hoboken.

[10] Flury, M., Merz, R., Le Boudec, J.-Y. and Zory, J. (2007) Performance Evaluation of an IEEE 802.15.4a Physical Layer with Energy Detection and Multi-User Interference. Book Performance Evaluation of an IEEE 802.15.4a Physical Layer with Energy Detection and Multi-User Interference, Series Performance Evaluation of an IEEE 802.1.

[11] Shen, G.W., Zetik, R., Hirsch, O. and Thomä, R.S. (2010) Range-Based Localization for UWB Sensor Networks in Realistic Environments. EURASIP Journal on Wireless Communications and Networking, 2010, Article ID: 476598.

[12] Richardson, P. and Shan, D. (2009) Experimental Data Collection and Performance Analysis of Outdoor UWB Positioning System under Static and Mobile Conditions. EURASIP Journal on Wireless Communications and Networking, 2009, Article ID: 618036.

[13] Zhang, H. and Gulliver, T. (2008) Capacity of Time-Hopping PPM and PAM UWB Multiple Access Communications over Indoor Fading Channels. EURASIP Journal on Wireless Communications and Networking, 2008, Article ID: 273018.

[14] Yang, H. and Yang, S.H. (2007) RFID Sensor Network-Network Architectures to Integrate RFID, Sensor and WSN. Measurement and Control, 40, 56-59.

[15] Liu, H., Bolic, M., Nayak, A. and Stojmenovic, I. (2008) Taxonomy and Challenges of the Integration of RFID and Wireless Sensor Networks. IEEE Network, 22, 26-35. http://dx.doi.org/10.1109/MNET.2008.4694171

[16] Win, M.Z. and Scholtz, R.A. (2000) Ultra-Wide Bandwidth Time-Hopping Spread-Spectrum Impulse Radio for Wireless Multiple-Access Communications. IEEE Transactions on Communications, 48, 679-689. http://dx.doi.org/10.1109/26.843135

[17] Ismail, W., Mandeep, J.S. and Jawad, M.S. (2008) Secure Multi-Access Channel Using UWB for Next Generation RFID Systems. Microwave Journal.

[18] Biradar, G.S., Merchant, S.N. and Desai, U.B. (2007) Adaptive Time Hopping PPM UWB Multiple Access Communication Schemes. International Symposium on Communications and Information Technologies, Sydney, 17-19 October 2007, 1258-1262.

[19] Sahin, M.E. and Arslan, H. (2005) Inter-Symbol Interference in High Data Rate UWB Communications Using Energy Detector Receivers. IEEE International Conference on Ultra-Wideband, 5-8 September 2005, 176-179.

[20] Ahmed, S. and Arslan, H. (2007) Inter-Symbol Interference in High Data Rate Transmit Reference UWB Transceivers. IEEE International Conference on Ultra-Wideband, Singapore, 24-26 September 2007, 773-778.

[21] Lottici, V., Wu, L. and Tian, Z. (2007) Inter-Symbol Interference Mitigation in High-Data-Rate UWB Systems. IEEE International Conference on Communications, Glasgow, 24-28 June 2007, 4299-4304.

[22] August, N.J., Thirugnanam, R. and Dong Sam, H. (2004) An Adaptive UWB Modulation Scheme for Optimization of Energy, BER, and Data Rate. Joint with Conference on Ultrawideband Systems and Technologies. Joint UWBST \& IWUWBS. 2004 International Workshop on Ultra Wideband Systems, 18-21 May 2004, 182-186. 
Scientific Research Publishing (SCIRP) is one of the largest Open Access journal publishers. It is currently publishing more than 200 open access, online, peer-reviewed journals covering a wide range of academic disciplines. SCIRP serves the worldwide academic communities and contributes to the progress and application of science with its publication.

Other selected journals from SCIRP are listed as below. Submit your manuscript to us via either submit@scirp.org or Online Submission Portal.
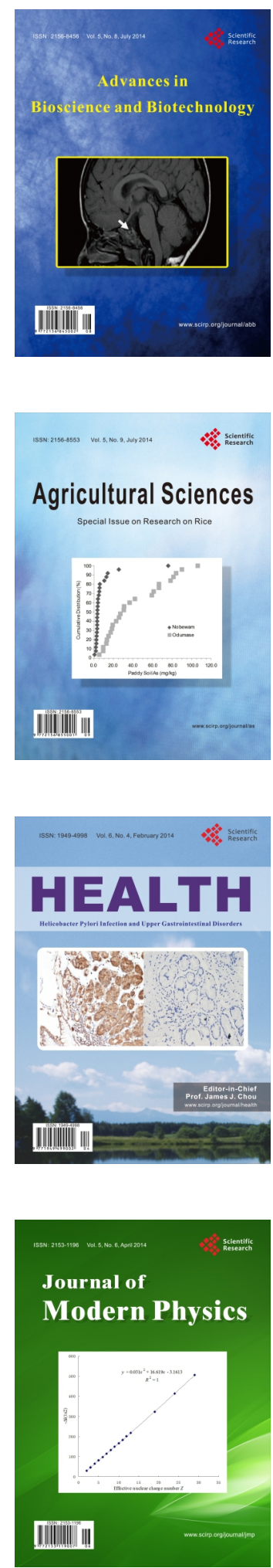
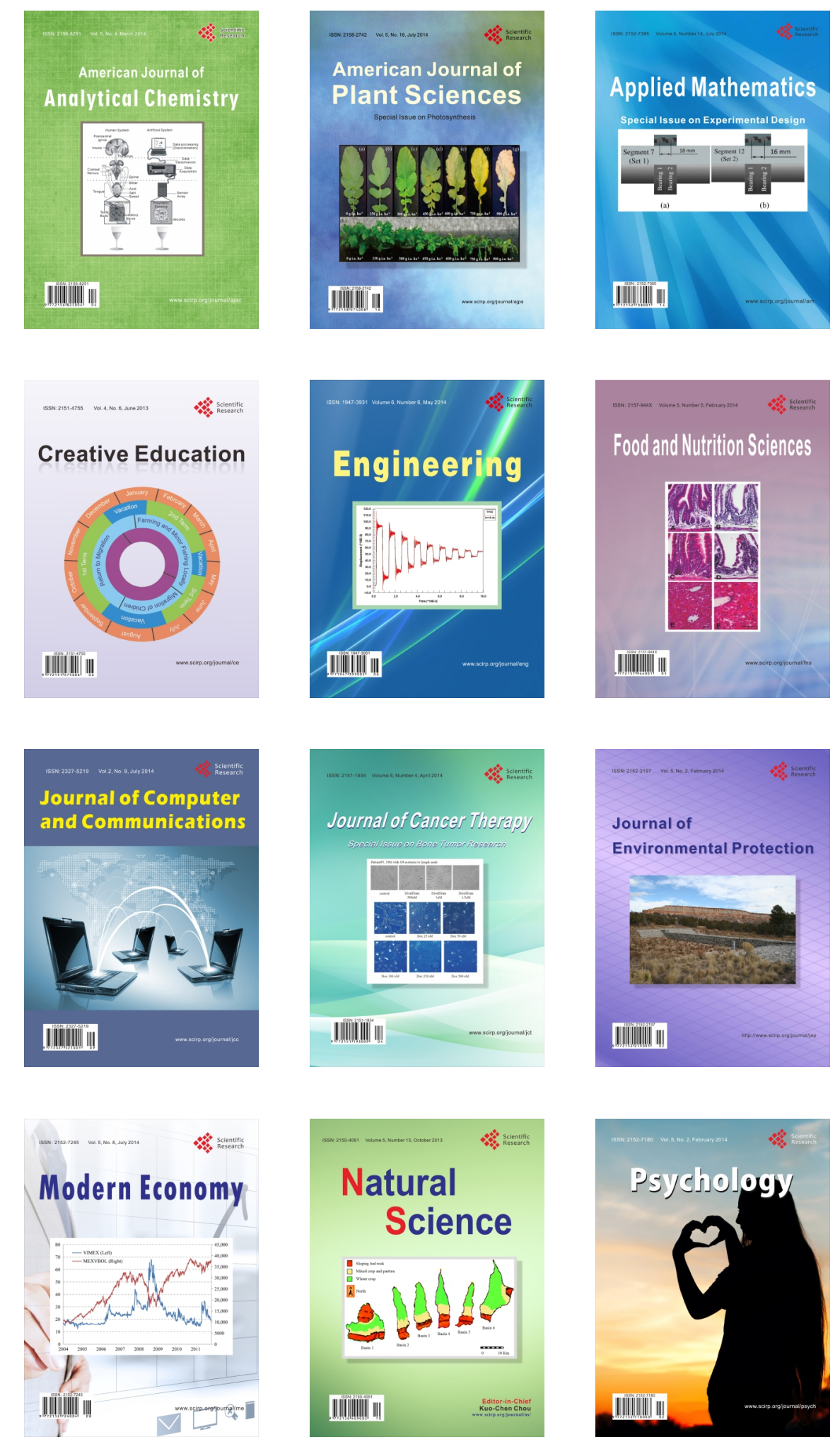